\title{
Tissue Engineering of Human Heart Valve Leaflets: A Novel Bioreactor for a Strain-Based Conditioning Approach
}

\author{
Anita Mol, ${ }^{1,2}$ Niels J. B. Driessen, ${ }^{1}$ Marcel C. M. Rutten, ${ }^{1}$ Simon P. Hoerstrup,, 2 \\ CARliJn V. C. Bouten, ${ }^{1}$ and Frank P. T. BaAiJENs ${ }^{1}$ \\ ${ }^{1}$ Eindhoven University of Technology, Department of Biomedical Engineering, Eindhoven, The Netherlands and ${ }^{2}$ University Hospital \\ Zürich, Clinic for Cardiovascular Surgery, Raemistrasse, Zürich, Switzerland
}

(Received 8 April 2005; accepted 16 August 2005)

\begin{abstract}
Current mechanical conditioning approaches for heart valve tissue engineering concentrate on mimicking the opening and closing behavior of the leaflets, either or not in combination with tissue straining. This study describes a novel approach by mimicking only the diastolic phase of the cardiac cycle, resulting in tissue straining. A novel, yet simplified, bioreactor system was developed for this purpose by applying a dynamic pressure difference over a closed tissue engineered valve, thereby inducing dynamic strains within the leaflets. Besides the use of dynamic strains, the developing leaflet tissues were exposed to prestrain induced by the use of a stented geometry. To demonstrate the feasibility of this strain-based conditioning approach, human heart valve leaflets were engineered and their mechanial behavior evaluated. The actual dynamic strain magnitude in the leaflets over time was estimated using numerical analyses. Preliminary results showed superior tissue formation and non-linear tissuelike mechanical properties in the strained valves when compared to non-loaded tissue strips. In conclusion, the strain-based conditioning approach, using both prestrain and dynamic strains, offers new possibilities for bioreactor design and optimization of tissue properties towards a tissue-engineered aortic human heart valve replacement.
\end{abstract}

Keywords-Heart valve prostheses, Bioreactor, Mechanical conditioning, Straining, Modeling.

\section{INTRODUCTION}

In cardiovascular tissue engineering, stimulation of tissue formation by mechanical conditioning has proven to be a useful tool for development of functional cardiovascular structures enabling growth, repair, and remodeling. The most common way of conditioning a developing tissue is by mimicking its physiological environment in vivo. Various bioreactor systems have been developed in the past years to apply specific conditioning protocols to growing cardiovascular structures.

Address correspondence to Anita Mol, Eindhoven University of Technology, Department of Biomedical Engineering, Laboratory for Biomechanics and Tissue Engineering, P.O. Box 513, Building W-hoog 4.112, 5600 MB Eindhoven, The Netherlands. Electronic mail: a.mol@tue.nl
Bioreactors developed for tissue engineering of heart valves all mimic the opening- and closing behavior of the heart valves in vivo. ${ }^{8,9}$ By using pulsatile medium flow through the developing valve, the systolic phase of the cardiac cycle is represented. The diastolic phase is represented when the valve leaflets are closed, due to minimal flow, and medium is resting onto the valve leaflets, inducing a pressure difference. Similar to heart valves, developing blood vessels are also conditioned by mimicking the full physiological environment. ${ }^{19,28,34}$ Pulsatile flow through the vessel lumen represents the blood stream and due to the pulsatile character of the flow, mimicking the cardiac output at every heartbeat, the blood vessel wall is strained. More complex systems, ensuring exact physiological conditions, have been developed for both blood vessels ${ }^{14,15,30}$ and heart valves ${ }^{5,7,23}$ and can be used for testing purposes as well.

Despite these efforts, the question still remains whether the developing tissue should indeed be exposed to all mechanical cues present in its physiological environment, or whether it is more beneficial to mimic its environment only partly. Tissue-engineered blood vessels, for example, were shown to grow and develop very well in bioreactors that only used dynamic wall straining, without the use of pulsatile flow. ${ }^{20,21,27}$ Culturing in an initial low shear stress environment combined with exposure of the developing tissue to physical stimuli has been suggested by Barron et al. ${ }^{1}$ as well to be advantageous for initial tissue development. In this study, the hypothesis of mimicking only the phase of the cardiac cycle in which the heart valve is most prone to dynamic straining, being beneficial for tissue growth and development, is tested. A prerequisite for the application of strains in this approach is to ensure a nonporous tissue structure. For this purpose, fibrin was used as a cell carrier. ${ }^{17}$ In the field of heart valve tissue engineering, this strain-based conditioning approach, without the use of pulsatile flow, has not been described and tested before.

In order to mimic solely the phase of the cardiac cycle where the tissue is being strained, a new bioreactor 
system is developed, the Diastolic Pulse Duplicator (DPD). In this system, dynamic strains are induced in the heart valve leaflets by applying a dynamic pressure difference over the closed valve leaflets. Requirements for such a bioreactor system are: (1) simplicity in its use, (2) sized small to save on culture medium and incubator space, (3) usage of biocompatible materials, (4) maintenance of sterility over prolonged periods of time, and (5) the ability to monitor and control the applied transvalvular pressure. Compaction-induced prestrain develops in the leaflets as tissue compaction, common in growing and healing tissues, is constrained by the use of a rigid stent. Theoretically, the amount of dynamic strains can be monitored using markers on the leaflets. As this is a time-consuming and in practice rather difficult method, we have chosen to estimate the range of dynamic strains in the leaflets by finite element analyses.

The cells in the tissue-engineered structures are responsible for the formation of the extracellular matrix and via mechanical conditioning they can be stimulated to produce larger amounts of extracellular matrix. Apart from the nature and magnitude of the mechanical cues, the cellular (pheno)type as well as the culture conditions (either $2 \mathrm{D}$ or in a $3 \mathrm{D}$ environment) affect the cellular responses to mechanical loading. ${ }^{2,3,6,10-13,22,32,33}$. In this study, human saphenous vein cells were chosen as a cell source for tissue engineering of human heart valve leaflets. Not only do they represent an easily accessible cell source, they were also shown to be more sensitive to mechanical cues compared to human arterial derived cells. ${ }^{3,25}$ Furthermore, increased extracellular matrix formation has been demonstrated in engineered heart valve leaflet tissue-equivalents cultured with these cells using dynamic strains. $^{16}$

The feasibility of the use of the DPD for human heart valve leaflet tissue engineering is demonstrated and preliminary results are presented, based on non-woven polyglycolic acid (PGA) meshes coated with poly-4hydroxybutyrate (P4HB). Tissue formation was analyzed qualitatively and mechanical properties quantitatively of engineered leaflets exposed to prestrain and additional dynamic strains and prestrain only and compared to unloaded tissue strips, serving as control.

\section{MATERIALS AND METHODS}

\section{Preparation of the Leaflet Tissues}

\section{The Heart Valve Scaffold}

Trileaflet heart valve scaffolds were fabricated on a Fastacryl $^{\circledR}$ stent. The two components, Fastacryl powder and fluid (PMMA and MMMA, Vertex-dental, the Netherlands) were mixed, poured into a mold, and allowed to polymerize for $30 \mathrm{~min}$. After complete polymerization, the stent was released from the mold. Anatomically shaped leaflets, including coaptation areas (Fig. 1(a)), were cut out of non-woven polyglycolic acid meshes (PGA; thickness $1.0 \mathrm{~mm}$; specific gravity $69 \mathrm{mg} / \mathrm{cm}^{3}$; Cellon, Luxembourg). The resulting stented valve leaflet geometry can be described using the parameters defined by Thubrikar: ${ }^{31}$ the angle of open leaflet $(\beta)=0$, radius of the commissures $(\mathrm{Rc})=$ radius of the base $(\mathrm{Rb})=11.5 \mathrm{~mm}$, and valve height $(\mathrm{H})$ minus height of the commissures $(\mathrm{Hs})=$ $13 \mathrm{~mm}$. The leaflets were coated with a thin layer of poly4-hydroxybutyrate (P4HB; MW $1 \times 10^{6}$; TEPHA Inc., Cambridge, USA) as described before. ${ }^{8}$ Before evaporation of the solvent, the leaflets were positioned onto a teflon mold in the shape of a trileaflet heart valve. The fastacryl stent was placed on top. By action of the solvent, dissolving the surface layer of the stent, the leaflets were fixed to the stent. After evaporation of the solvent, the valve scaffold including the stent was removed from the mold (Fig. 1(b) and (c)). The valve scaffolds were sterilized using ethylene oxide.

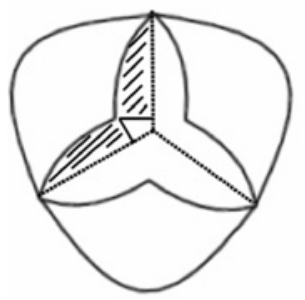

(a)

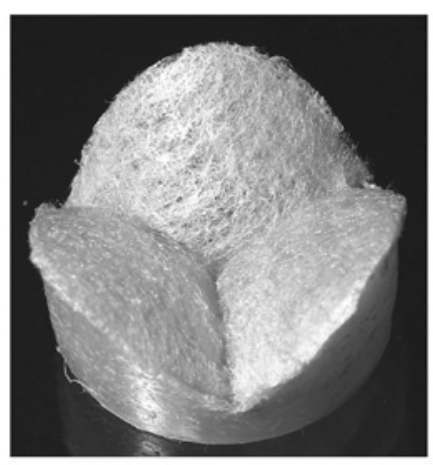

(b)

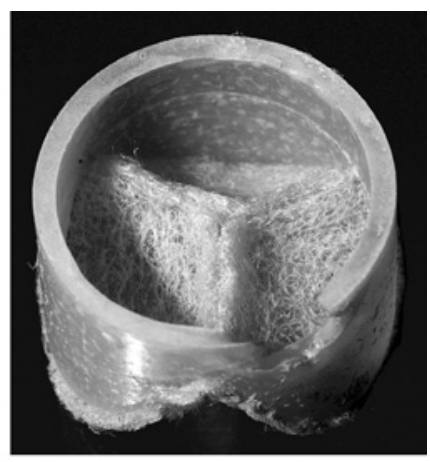

(c)

FIGURE 1. Two-dimensional valve leaflet geometry (a) and photographs of the stented valve scaffold, bottomview (b) and topview (c). The scaffold is fabricated from a non-woven PGA mesh, coated with P4HB and includes a fastacryl stent fixed to the leaflets. The initial circumferential size of the leaflets is $23 \mathrm{~mm}$. The leaflets contain coaptation areas to ensure closure of the valve. 
Seeding Procedure

Cells harvested from the human vena saphena magna and expanded using regular cell culture methods were used. ${ }^{25}$ The medium to culture these cells consisted of DMEM Advanced (Gibco, USA), supplemented with 10\% Fetal Bovine Serum (FBS; PAN Biotech, Germany), 1\% GlutaMax (Gibco, USA), and $0.1 \%$ gentamycin (PAN Biotech, Germany). The medium used for seeding and subsequent tissue culture contained $0.3 \%$ gentamycin and additional L-ascorbic acid 2-phosphate $(0.25 \mathrm{mg} / \mathrm{ml}$; Sigma, USA) to promote extracellular matrix production. The scaffolds were placed in medium overnight before seeding to facilitate cell attachment by deposition of proteins. The seeding was performed per leaflet using fibrin as a cell carrier. ${ }^{17}$ Briefly, the cells were suspended in a sterile thrombin solution (10 IU/ml medium; Sigma, USA) in a volume that equals half the void volume of the scaffold $(0.5 \times$ length $\times$ width $\times$ thickness). The cells in thrombin were mixed with an equal amount of sterile fibrinogen solution $(10 \mathrm{mg}$ actual protein/ml medium; Sigma, USA) and dripped onto the scaffold. The fibrin solution was taken up by the scaffold and remained inside due to polymerization of the fibrin gel. The leaflets were seeded with a density of 4-5 million cells (passage 6-7) per $\mathrm{cm}^{2}$ of scaffold. The seeded valve scaffolds were allowed to polymerize for $20 \mathrm{~min}$ in an incubator $\left(37^{\circ} \mathrm{C}\right.$ and $5 \% \mathrm{CO}_{2}$ ) before placement into the DPD.

\section{The Diastolic Pulse Duplicator (DPD)}

\section{Description of the DPD}

Each DPD consists of two components as shown in Fig. 2(a): a bioreactor (height $=9 \mathrm{~cm}$, diameter $=6 \mathrm{~cm}$ ), in which the valve is cultured, and a medium container of similar dimensions, both fabricated from polycarbonate (KUBRA Kunststoffen, The Netherlands). The bioreactor itself consists of two parts, the upper part containing a glass window (Melles Griot BV, The Netherlands) for visualization of the valve, and a lower part, which are screwed together. Silicone rings (van der Heijden, The Netherlands) are used to seal all components. The bioreactor and the medium container are connected via two parallel silicone tubing series (Rubber, The Netherlands). Polypropylene connector parts (Neolab, Milispec Int., The Netherlands) are used to secure the tubing. Both tubing series run through a roller pump (Masterflex, ${ }^{\circledR}$ Cole-Parmer, USA). Part of the upper tubing series consists of a thicker silicone tube placed in a polycarbonate cylinder, with a connection to tubing suitable to withstand air under pressure (Festo, The Netherlands). This compressed-air tubing is connected to a compressed-air tap ( 7 bar). The air pressure is reduced to 2 bar and runs through a proportional magnet valve (Festo, The Netherlands) into the polycarbonate cylinder. The complete DPD is sterilized by ethylene oxide and is placed inside an incubator, together with the roller pump. Up to six

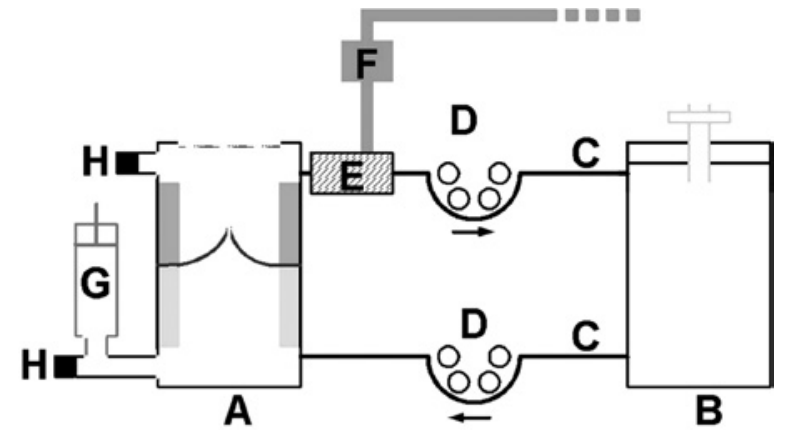

(a)

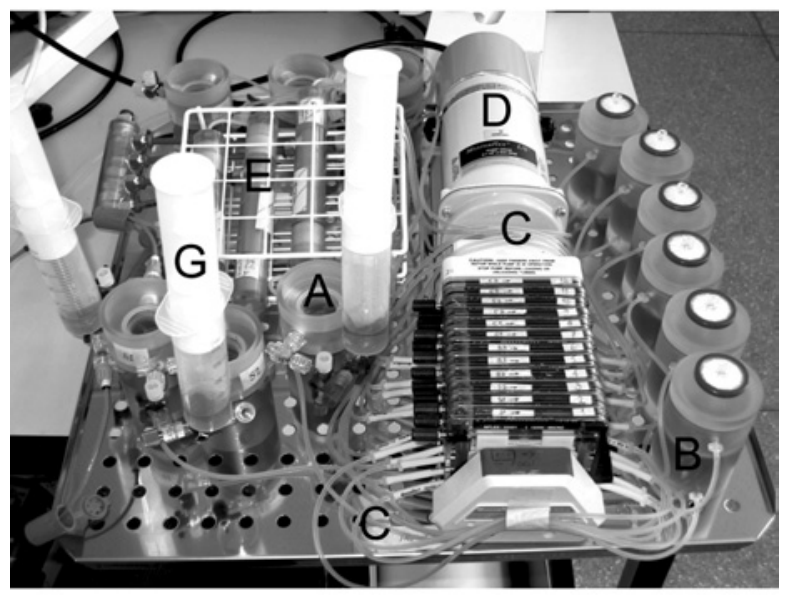

(b)

FIGURE 2. Schematic drawing of one DPD and its function (a) and a photograph of six DPD systems in use simultaneously (b). One DPD consists of a bioreactor (A), in which the valve is cultured, and a medium container (B). They are connected to each other via two parallel tubing series (C), which run through a roller pump (D). Part of the upper tubing consists of a thicker tube placed in a polycarbonate cylinder (E). Compressed air can be released into this cylinder, via a magnet valve $(F)$, to compress and decompress the tubing, resulting in a pressure difference over the leaflets. A syringe (G), placed on the bioreactor, serves as a compliance chamber. The pressures upstream and downstream of the leaflets is monitored using pressure sensors $(\mathrm{H})$.

DPDs are placed onto one shelf of a normal sized incubator as shown in Fig. 2(b).

\section{Functioning of the DPD}

About $75 \mathrm{ml}$ of medium is circulating from the medium container, through the bioreactor, and back into the medium container, via the roller pump at very low speed $(4 \mathrm{ml} / \mathrm{min})$ to supply fresh nutrients to and to remove waste products from the developing valve tissue. A sterile filter $(0.2 \mu \mathrm{m}$, Schleicher \& Schuell Bioscience, Germany) is placed in the lid of the medium container to oxygenate the circulating medium. About $25 \mathrm{ml}$ of medium is present in the bioreactor and the tubing series, and the remaining $50 \mathrm{ml}$ is present in the medium container and can be changed 
easily. To apply a dynamic pressure difference over the leaflets, the silicone tube in the polycarbonate cylinder is compressed and decompressed by the air coming from the proportional magnet valve. The transvalvular pressure generated over the valve leaflets is controlled via a programmable multi-IO-card using Lab View software (National Instruments, USA). The shape and frequency of the pressure wave is programmed for an optimal transvalvular pressure. A syringe, connected to the lower part of the bioreactor, filled with $10 \mathrm{ml}$ of medium and $40 \mathrm{ml}$ of air, serves as compliance chamber. Pressure sensors (BD, Belgium), connected to both the lower and upper part of the bioreactor, are used to measure the pressure upstream (at the ventricular side) and downstream (at the arterial side) of the leaflets. Using the same multi-IO-card and software, the dynamic pressure difference is monitored and logged.

\section{Sterility and Biocompatibility}

To prove maintained sterility over several weeks, the DPD was sterilized by ethylene oxide and filled with $75 \mathrm{ml}$ of culture medium without the addition of gentamycin. The system was fully functional for a period of 3 weeks. During the testing period, a culture flask filled with culture medium, without gentamycin, served as control. The medium in the medium container was replaced every 3-4 days as well as two thirds of the medium in the culture flask. The medium was checked microscopically for contamination and was stored at $-20^{\circ} \mathrm{C}$ until further use.

In order to ensure that the materials used in the DPD are suitable for cultivation of heart valves and that no toxic components are being released, a biocompatibility test was performed using the medium that was stored during the sterility test. Human saphenous vein cells were seeded into 24 wells plates (30.000 cells/well) and left overnight to attach and spread in an incubator in normal cell culture medium. The next day, the normal cell culture medium was replaced by the medium of the sterility test that had circulated through the DPD and the control medium (5 wells per test group). The medium stored after the first four medium changes, as well as the control medium from the culture flask, was used. The cells were allowed to grow for three subsequent days, after which the metabolic ac- tivity of the cells, as a measure for viability, was determined using an MTT test. ${ }^{18}$ Briefly, MTT (Sigma, USA) in solution $(5 \mathrm{mg} / \mathrm{ml}$ in PBS) was diluted in medium and added to the cells. After one hour of incubation at $37^{\circ} \mathrm{C}$ and $5 \% \mathrm{CO}_{2}$, metabolic active cells had converted the MTT salt into purple crystals located inside the cells. Isopropanol (VWR International, USA), containing 10\% formic acid (Sigma, USA) was added to the cells to release and dissolve the purple crystals and the optical density of the solution was determined. The results are expressed as percentages with respect to the control, which was set at $100 \%$.

\section{Tissue Culture and Mechanical Conditioning}

To demonstrate the feasibility of the strain-based conditioning approach, a preliminary experiment was performed. Three groups of valve leaflet tissues were engineered $(n=3$ per group, Table 1) to compare tissue formation and mechanical properties. The first group comprises rectangular shaped tissue strips. They were cultured statically in a cell culture flask for up to 4 weeks and could compact freely, serving as non-loaded controls. The second and third group consisted of valve leaflets, all cultured in the DPD. Directly after placement in the DPD, all leaflets were exposed to medium circulation at low speed $(4 \mathrm{ml} / \mathrm{min})$ to supply fresh nutrients to the developing tissue. Valve leaflets were cultured up to 4 weeks, exposed to continuous medium circulation and prestrain due to compaction constrained by the stent (group 2). Additional valve leaflets were exposed to dynamic strains at $1 \mathrm{~Hz}$ in addition to the prestrain and continuous medium circulation (group 3). The pressures upstream and downstream of the valves, as well as the dynamic transvalvular pressure, were recorded every three hours. The valve leaflets of group 3 were cultured up to 2 , 3 , and 4 weeks. The medium in the medium container, as well as two thirds of the medium in the culture flask for group 1, was replaced every 3-4 days.

\section{Evaluation of Tissue Formation}

\section{Histology}

Tissue formation in all groups was analyzed by histology after 4 weeks. Representative pieces were fixed in phosphate-buffered formalin (Fluka, USA) and embedded

TABLE 1. Overview of the experimental groups: (1) non-loaded tissue strips, (2) leaflets exposed to prestrain in the DPD, and (3) leaflets exposed to prestrain and additional dynamic strains in the DPD.

\begin{tabular}{clllll}
\hline Group no & Geometry & Culture conditions & Mechanical conditioning & Culture time & Number of leaflets $(n)$ \\
\hline 1 & Strips & Static in flask & None & 4 weeks & 3 \\
2 & Leaflets & Medium circulation in DPD & Prestrain & 4 weeks & 3 \\
3 & Leaflets & $\begin{array}{c}\text { Medium circulation and } \\
\text { dynamic strains in DPD }\end{array}$ & Prestrain and dynamic strain & 2 , 3, and 4 weeks & 3 per time point \\
& & & & \\
\hline
\end{tabular}

Note. An $n$ of 1 comprises a single engineered leaflet tissue. 
in paraffin. Sections were cut at $5 \mu \mathrm{m}$ thickness and studied by Haematoxylin and Eosin (H\&E) staining for general tissue morphology and Trichrome Masson staining for collagen formation, as collagen is the main load-bearing component of the extracellular matrix.

\section{Mechanical Tests}

The mechanical properties of circumferential strips of the engineered leaflet tissues of the three groups after 4 weeks of culturing were determined by uniaxial tensile tests. As thickness measurements of the fresh strips were practically difficult, the thickness was determined from representative histology sections. A tissue volume shrinkage correction factor of 1.15 (1.046 linear shrinkage) was used to correct for the supposed loss of tissue volume due to tissue processing. ${ }^{24}$ Stress-strain curves were obtained using an uniaxial tensile tester (Instron, Belgium, model 4411, equipped with a load cell of $10 \mathrm{~N}$ ) with a constant strain rate of $1.7 \%$ per second. From the stress-strain curves, the ultimate tensile strength (UTS) was determined, as well as the strain at break. The modulus was calculated, defined as the slope of the linear portion of the curve. To get insight into the evolution of mechanical properties in time, the mechanical properties of circumferential strips of dynamically strained leaflets after 2 and 3 weeks of culturing were additionally determined.

\section{Statistics}

Results of the mechanical tests are expressed as mean \pm standard error of the mean. One-way ANOVA analyses were used to determine significant differences between the tested groups, followed by Tukey HSD post-hoc tests between groups (STATGRAPHICS Plus 5.1, Statistical Graphics Corp., USA) in case of a significant result. Differences were considered significant at a $p$-value $<0.05$.

\section{Estimation of Strains in the Leaflets}

\section{Prestrain}

To estimate the amount of prestrain in the leaflets, due to compaction constrained by the stent, the difference in size of the leaflets in the stent after culturing and after release from the stent was determined. The leaflets, having an initially bulged shape in the stent, did straighten during culturing due to free compaction of the neo-tissue. The initial circumferential size of the leaflets was $23 \mathrm{~mm}$ (Fig. 1(a)). This circumferential size was reduced to $20 \mathrm{~mm}$ by straightening of the leaflets in the stent during culturing (Fig. 3(a)), indicating that the leaflets could compact freely up to $13 \%$ during culturing. The circumferential size of each leaflet was measured after release from the stent (Fig. 3(b)) of the valves exposed to prestrain and medium circulation after 4 weeks. This measured size was divided

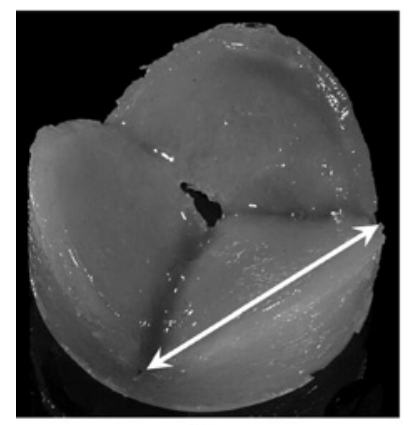

(a)

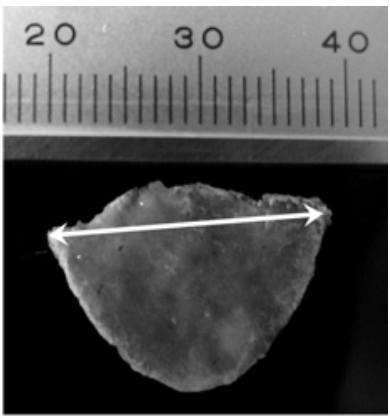

(b)
FIGURE 3. The circumferential size of the fully flattened leaflets in the stent after culturing (a) was $20 \mathrm{~mm}$. After release of the leaflets from the stent (b), the circumferential size was measured and the difference in size was used to calculate the amount of prestrain in the leaflets during culturing.

by the maximally straightened size of $20 \mathrm{~mm}$ to calculate the amount of prestrain in the leaflets. This value was subsequently extracted from 1 and multiplied by $100 \%$ to obtain the amount expressed in a percentage of prestrain.

\section{Dynamic Strains}

Finite element analyses were used to estimate the amount and distribution of the dynamic strains in the leaflets resulting from the applied dynamic transvalvular pressure. The finite element mesh of the stented valve geometry is shown in Fig. 4. The configuration shown in this figure was assumed to be stress-free and because of symmetry only $1 / 2$ of a valve leaflet was used in the finite element analyses. At the symmetry edge, nodal displacements in the normal direction were suppressed, whereas at the fixed edge all nodal displacements were set to zero. At the free edge, a contact surface was defined to model coaptation of adjacent leaflets. The transvalvular pressure $p_{t v}$ was subsequently

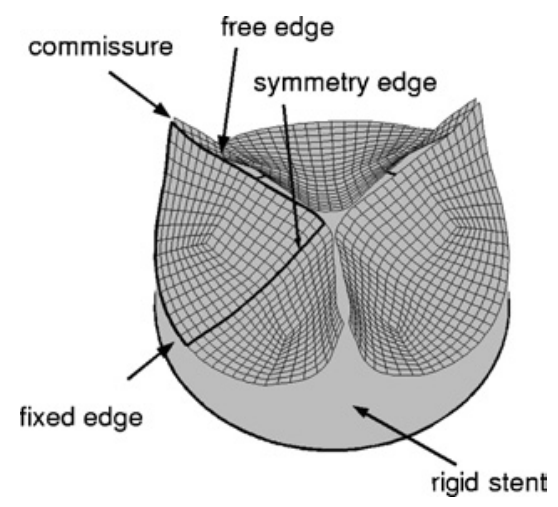

FIGURE 4. Finite element mesh of the stented valve geometry. Because of symmetry only $1 / 2$ of a leaflet is used in the finite element analyses. This part of the geometry is discretized using 224 hexahedral elements. 
applied to the downstream surface of the leaflets. In this study, the finite element package SEPRAN was used. ${ }^{26}$

The valve tissues were modeled as an incompressible generalized Neo-Hookean material:

$$
\boldsymbol{\sigma}=-p \boldsymbol{I}+G(\boldsymbol{B}-\boldsymbol{I})
$$

with $\sigma$ the Cauchy stress, $p$ the hydrostatic pressure, $I$ the unity tensor, and $G$ the shear modulus of the material. The left Cauchy-Green deformation tensor was defined as $\boldsymbol{B}=\boldsymbol{F} \cdot \boldsymbol{F}^{T}$ with $\boldsymbol{F}$ the deformation gradient tensor. To describe the (potential) non-linear behavior of the leaflet tissues, the following expression for $G$ was used:

$$
G=G_{0}\left(\frac{I_{1}(\boldsymbol{B})}{3}\right)^{n}
$$

with $G_{0}$ and $n$ material parameters and $I_{1}(\boldsymbol{B})=\operatorname{trace}(\boldsymbol{B})$ the first invariant of $\boldsymbol{B}$. The parameter $n$ is used to control the degree of non-linearity of the constitutive equation: $n>0$ indicates stiffening of the material with increasing stretches, whereas $n<0$ indicates softening. Note that the classical Neo-Hookean model is obtained for $n=0$, with $G=G_{0}$. The material parameters were obtained by fitting the constitutive law (Eq. (1)) to the mean results before failure of the uniaxial tensile tests of the leaflet tissues exposed to dynamic strains after 2, 3, and 4 weeks of culturing.

\section{RESULTS}

\section{The Diastolic Pulse Duplicator}

The medium, without addition of antibiotics, which had circulated in the DPD did not show any macroscopic or microscopic signs of contamination for the complete test period. The system was easy to handle and the risk of contamination during medium replacement was minimal. The results of the biocompatibility test, performed for the first four medium changes during the sterility test, are shown in Fig. 5. The metabolic activity of the cells cultured with the four tested media were all within 80-100\% when compared to the controls, indicating the DPD to be biocompatible and suitable for cultivation of human heart valves.

\section{Dynamic Straining Protocol}

A representative transvalvular pressure curve, measured during culturing, is shown in Fig. 6. The permanent transvalvular pressure, present over the valve leaflets, is referred to as the DC offset, which is the average value of the difference in pressure upstream and downstream of the leaflets. The cyclic transvalvular pressure is defined as the peak-to-peak transvalvular pressure value. The maximum transvalvular pressure value, including the DC offset and

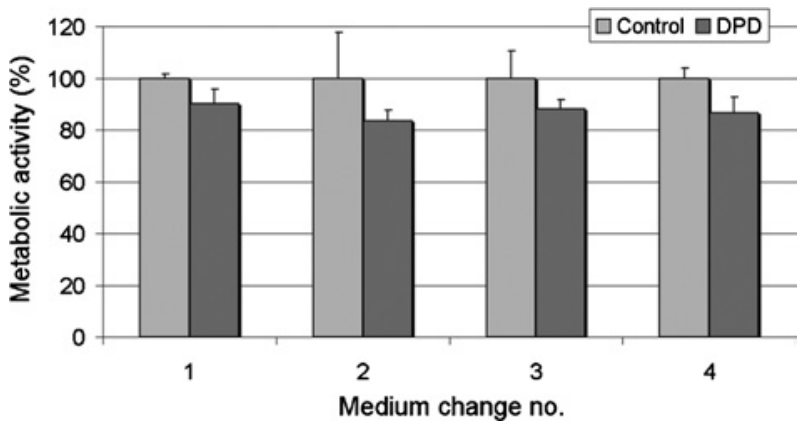

FIGURE 5. Metabolic activity of cells cultured with the first four changes of the medium that had circulated in the DPD during the sterility test. The tested medium (dark gray bars) was compared to control medium (light gray bars), which was set at $100 \%$. The metabolic activity was in all cases $80-100 \%$ compared to the controls and, therefore, the DPD can be considered as being biocompatible. The error bars represent the standard error of the mean.

the cyclic transvalvular pressure, is referred to as the applied dynamic transvalvular pressure.

The dynamic transvalvular pressure was averaged for each day, resulting in dynamic transvalvular pressures increasing gradually from 0 to about $80 \mathrm{~mm} \mathrm{Hg}$ within the first 2 weeks of culturing $(10 \mathrm{~mm} \mathrm{Hg}$ at day $7,30 \mathrm{~mm} \mathrm{Hg}$ at day $9,70 \mathrm{~mm} \mathrm{Hg}$ at day 12 , and $80 \mathrm{~mm} \mathrm{Hg}$ at day 14). During the last 2 weeks of culturing, the pressures were lowered due to the expected loss of support function of the scaffold, and kept constant at about $37 \mathrm{~mm} \mathrm{Hg}$.

\section{Evaluation of Tissue Formation}

\section{Histology}

Sections stained with H\&E and Trichrome Masson (Fig. 7) showed superior tissue formation in the leaflets

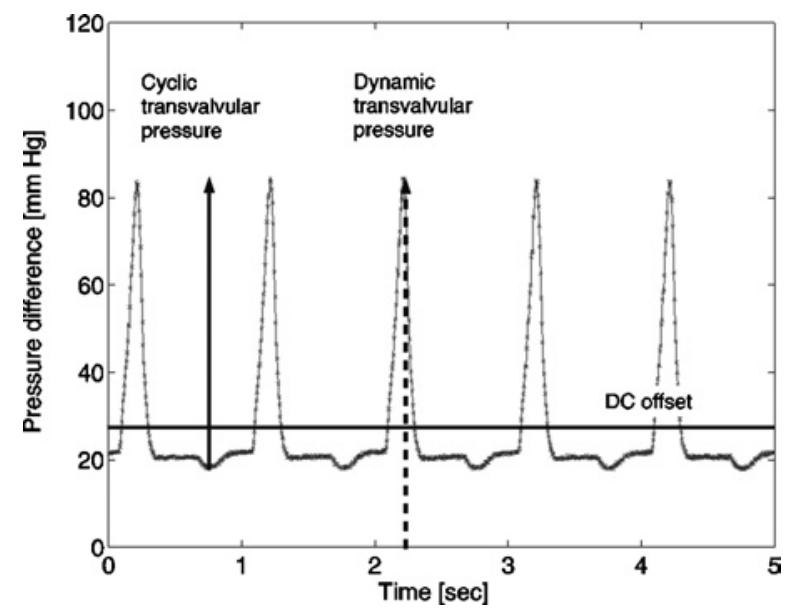

FIGURE 6. A representative transvalvular pressure curve measured during culturing of the valve leaflets. The definitions of DC offset, cyclic transvalvular pressure difference, and dynamic transvalvular pressure difference are represented. 


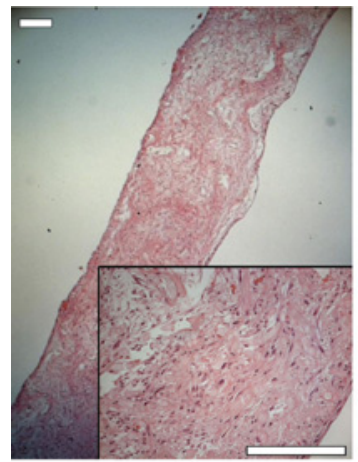

(a)

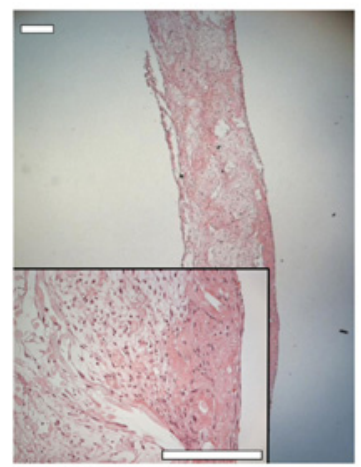

(c)

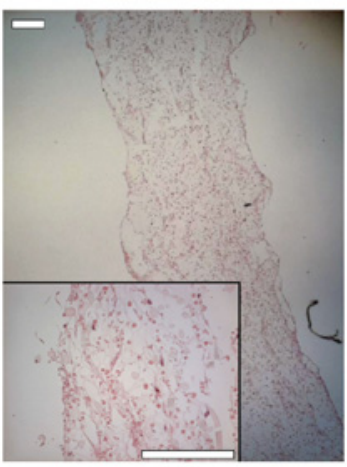

(e)

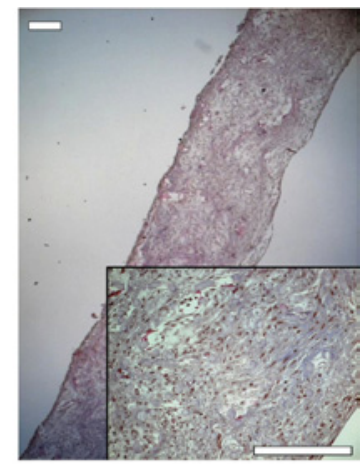

(b)

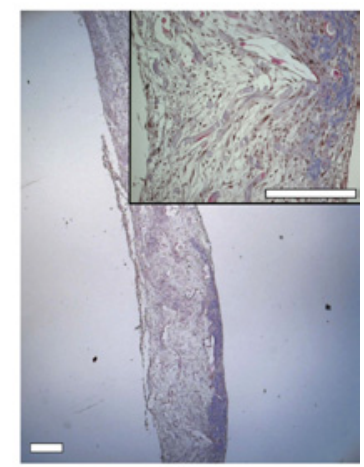

(d)

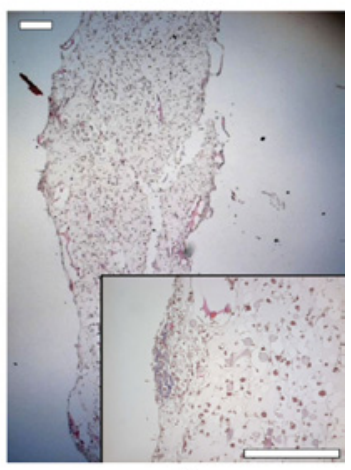

(f)
FIGURE 7. Representative sections of the valve leaflet tissues after 4 weeks of culturing stained with H\&E $(a, c, e)$ and Trichrome Masson (b,d,f). The bars in the images represent scales of $175 \mu \mathrm{m}$. The dynamically strained leaflets $(a, b)$ and the leaflets exposed to prestrain only $(c, d)$ showed superior tissue development when compared to the non-loaded valve leaflet tissue-equivalents (e,f). The dynamically strained leaflet tissue seemed more homogeneous and denser as compared to the leaflets exposed to prestrain only. Collagen, stained blue, could be identified in the leaflets $(b, d)$.

(Fig. 7(a)-(d)) when compared to the non-loaded rectangular leaflet tissue-equivalents (Fig. 7(e) and (f)). The tissue of the leaflets cultured with additional dynamic strains (Fig. 7(a) and (b)) appear to be more homogeneous and denser when compared to the leaflets exposed to prestrain only (Fig. 7(c) and (d)). Collagen could be identified in the leaflets, either cultured using prestrain or additional dynamic strains, after 4 weeks of culturing as shown in Fig. 7(d) and (b).

\section{Mechanical Tests}

The obtained stress-strain curves were averaged until failure per group and timepoint (Fig. 8). The values for UTS, modulus, and strain at break are summarized in Table 2. After 4 weeks, both leaflet types, either cultured using prestrain alone or using additional dynamic strains, were significantly stronger $(0.25 \pm 0.02 \mathrm{MPa}$ and $0.22 \pm$ $0.04 \mathrm{MPa}$, respectively) and stiffer $(1.03 \pm 0.03 \mathrm{MPa}$ and $0.78 \pm 0.20 \mathrm{MPa}$, respectively) when compared to the nonloaded tissue strips (UTS: $0.07 \pm 0.03 \mathrm{MPa}$, modulus:

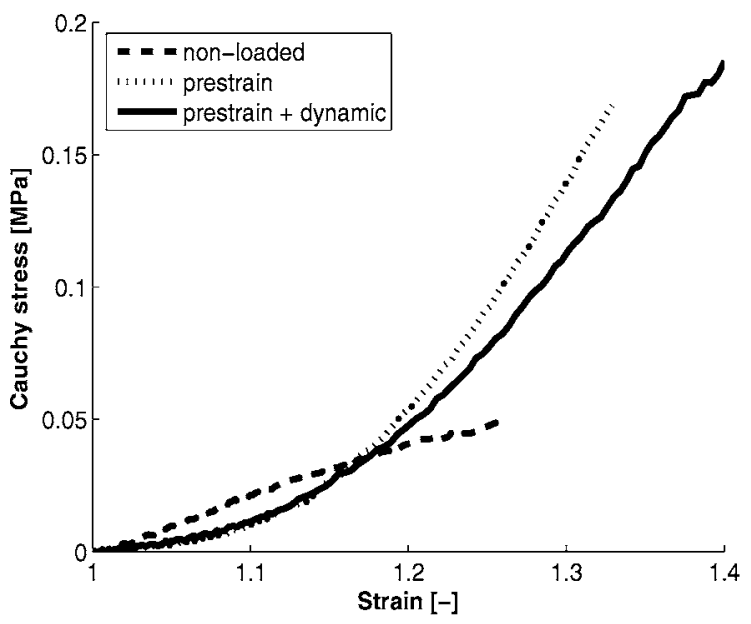

(a)

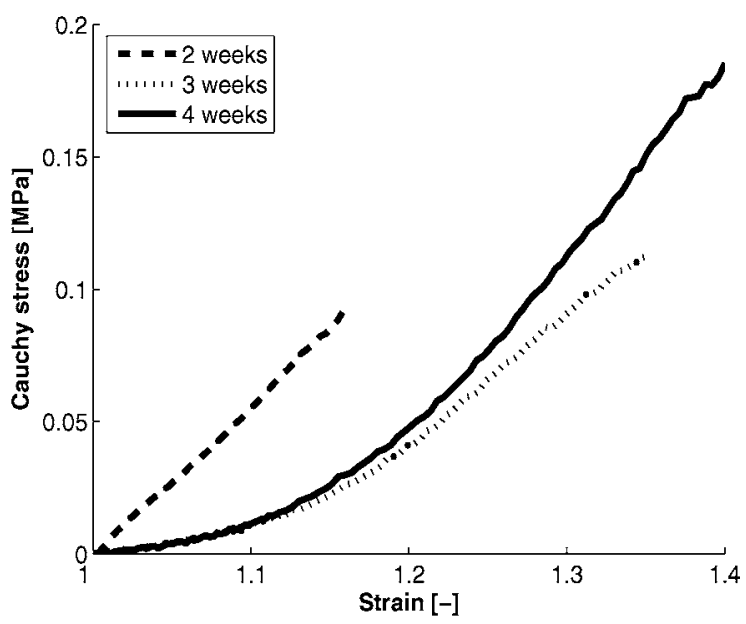

(b)

FIGURE 8. Stress-strain curves averaged before failure per group and timepoint, showing the mechanical behavior of nonloaded tissue strips, leaflets exposed to prestrain, and leaflets exposed to prestrain and additional dynamic strains after 4 weeks (a) and the evolution of mechanical properties over time for dynamically strained leaflets (b). The non-loaded tissue strips showed linear mechanical behavior, while the leaflet tissues showed tissue-like non-linear behavior after 4 weeks of culturing (a). Dynamic straining of the leaflet tissues during culturing resulted in more non-linear tissue-like mechanical behavior with time (b). 
TABLE 2. Summary of the results of the uniaxial tensile tests per group and per timepoint. The values are represented as average value \pm standard error of the mean.

\begin{tabular}{lcccc}
\hline \multicolumn{1}{c}{ Group } & Timepoint (weeks) & UTS (MPa) & Modulus (MPa) & Strain at break (\%) \\
\hline Prestrain and dynamic strains & 2 & $0.11 \pm 0.02$ & $0.66 \pm 0.08$ & $19 \pm 2$ \\
Prestrain and dynamic strains & 3 & $0.12 \pm 0.03$ & $0.53 \pm 0.14$ & $42 \pm 3^{\#}$ \\
Prestrain and dynamic strains & 4 & $0.22 \pm 0.04^{*}$ & $0.78 \pm 0.20^{*}$ & $44 \pm 1^{\#}$ \\
Prestrain & 4 & $0.25 \pm 0.02^{*}$ & $1.03 \pm 0.03^{*}$ & $41 \pm 4$ \\
Non-loaded strips & 4 & $0.07 \pm 0.03$ & $0.18 \pm 0.07$ & $46 \pm 11$ \\
\hline
\end{tabular}

Note. Shown are the ultimate tensile strength (UTS), the modulus, and the strain at break. Significant differences between the groups after 4 weeks of culturing are indicated $\left(^{*}, p<0.05\right)$ and shows the strips to be weaker and less stiff when compared to both types of leaflets. Significant differences between the timepoints are indicated (\#, $p<0.05)$ and shows an increase in strain at break after 3 and 4 weeks of culturing.

$0.18 \pm 0.07 \mathrm{MPa})$. No differences were found between leaflets cultured using prestrain and using additional dynamic strains. The non-loaded tissue strips showed linear mechanical behavior while the leaflets exposed to compaction-induced prestrain, either with or without additional dynamic strains, showed more tissue-like non-linear behavior (Fig. 8(a)).

With respect to the evolution of the mechanical properties over time of the dynamically strained leaflets, no significant increases in UTS $(0.11 \pm 0.02 \mathrm{MPa}$ after 2 weeks, $0.12 \pm 0.03 \mathrm{MPa}$ after 3 weeks, and $0.22 \pm 0.04 \mathrm{MPa}$ after 4 weeks $)$ and modulus $(0.66 \pm 0.08 \mathrm{MPa}$ after 2 weeks, $0.53 \pm 0.14 \mathrm{MPa}$ after 3 weeks, and $0.78 \pm 0.20 \mathrm{MPa}$ after 4 weeks) were identified. Strain at break increased after 3 and 4 weeks $(19 \pm 2 \%$ after 2 weeks, $42 \pm 3 \%$ after 3 weeks, and $44 \pm 1 \%$ after 4 weeks). It was evident that after 2 weeks of culturing the mechanical behavior was linear, representing scaffold-like behavior. After 3 and 4 weeks, the tissue showed more non-linear mechanical behavior, representative for tissue contribution (Fig. 8(b)).

\section{Estimation of Strains in the Leaflets}

\section{Prestrain}

The leaflets were fully straightened in the stent (Fig. 3(a)) and (partly) grown together. After releasing the leaflets from one another, the leaflets retracted some, caused by the prestrain in the leaflets, resulting in the loss of coaptation. The amount of prestrain in the leaflets of the valves exposed to prestrain only after 4 weeks of culturing, assuming maximal straightening of the leaflets, was $3.7 \pm 0.8 \%$ (mean value \pm standard error of the mean).

\section{Dynamic Strains}

The constitutive law (Eq. (1)) fitted the results of the uniaxial tensile tests quite reasonable. Table 3 summarizes the input parameters for the finite element model, the mean absolute error of the fits, and the estimated mean dynamic strains after 2, 3, and 4 weeks of culturing, based on a transvalvular pressure difference of $37 \mathrm{~mm} \mathrm{Hg}$. The mean dynamic strains in the leaflets increased from $8 \pm 4 \%$ after 2 weeks to $20 \pm 7 \%$ after 4 weeks of culturing within one leaflet. The dynamic strain distribution in the leaflets after 4 weeks of culturing is shown in Fig. 9, with in Fig. 9(a) and (b) the strain distribution within one leaflet at the upstream and downstream surface, respectively. Fig. 9(c) shows the estimated range of overall dynamic strains within a leaflet by means of a histogram.

\section{DISCUSSION}

Mechanical stimulation of tissue formation in a bioreactor system is a well-known technique in tissue engineering

TABLE 3. Summary of the input parameters for the finite element analyses of the dynamically strained leaflets after 2,3 , and 4 weeks of culturing.

\begin{tabular}{cccccc}
\hline $\begin{array}{c}\text { Culture time } \\
\text { (weeks) }\end{array}$ & $\begin{array}{c}\text { Thickness } \\
(\mathrm{mm})\end{array}$ & $\begin{array}{c}\text { Go } \\
(\mathrm{kPa})\end{array}$ & $n(-)$ & $\begin{array}{c}\text { Mean absolute } \\
\text { error of fit }(\mathrm{kPa})\end{array}$ & $\begin{array}{c}\text { Mean dynamic } \\
\text { strain }(\%)\end{array}$ \\
\hline 2 & 0.80 & 188 & 0.0 & 0.95 & $8 \pm 4$ \\
3 & 0.59 & 51 & 8.6 & 3.93 & $24 \pm 8$ \\
4 & 0.63 & 59 & 9.2 & 4.61 & $20 \pm 7$ \\
\hline
\end{tabular}

Note. The parameter $n$ was set to zero for the leaflets after 2 weeks of culturing, due to the observed linear behavior. Furthermore, the mean absolute errors of the fits of the constitutive law (Eq. (1)) to the results of the uniaxial tensile tests and the resulting estimated mean dynamic strains are represented as mean value \pm SD within one leaflet. 
of cardiovascular structures to improve tissue formation and organization. In this study, a novel bioreactor for tissue engineering of human heart valve leaflets is developed and preliminary results are presented to demonstrate its feasibility. Instead of mimicking the full cardiac cycle, only the phase in which the leaflets are most prone to dynamic strain (diastole) is mimicked in this bioreactor, the Diastolic Pulse Duplicator (DPD, Fig. 2). In the DPD, stented valve leaflets are exposed to continuous medium circulation at low speed $(4 \mathrm{ml} / \mathrm{min})$ to provide fresh nutrients to the tissue and to remove waste products. The leaflets are exposed to prestrain, induced by tissue compaction constrained by the stent. Dynamic strains are additionally applied in the DPD using a dynamic pressure difference over the leaflets. The DPD is very easy to handle, sized small with a total medium volume of only $75 \mathrm{ml}$ per valve, proven to be biocompatible (Fig. 5), and sterility could be maintained over prolonged periods of time.

To show the feasibility of the use of the DPD and the strain-based conditioning approach, three groups of leaflet tissues were engineered (Table 1), based on human saphenous vein cells, seeded using fibrin as a cell carrier onto PGA/P4HB scaffolds (Fig. 1). Non-loaded tissue strips showed lower mechanical properties and less tissue formation after 4 weeks of culturing when compared to the leaflets, which were exposed to prestrain only or to prestrain and additional dynamic strains (Table 2, Fig. 7). The mechanical behavior of both leaflet types was clearly nonlinear after 4 weeks of culturing, while the tissue strips showed linear behavior (Fig. 8(a)). The tissue of the leaflets exposed to dynamic strains appeared to be more homogeneous and denser packed as compared to leaflets exposed to prestrain only, however, this concerned only qualitative observation by histology. The mechanical properties were not significantly different for the two groups. Over time, the leaflets exposed to dynamic strains showed more nonlinear tissue-like behavior (Fig. 8(b)), indicating increasing amounts of tissue and collagen. The mechanical properties of the tissues engineered in this study approached those of native human aortic heart valve leaflets in radial direction. The circumferential mechanical properties of native leaflets are about 10 times higher. ${ }^{29}$ The continuous medium circulation in the DPD most likely contributes to the improved tissue formation in the leaflets. This contribution will be elucidated in future studies as well as possibilities to monitor tissue development during culturing by the identification of biochemical markers in the circulating medium. Furthermore, the application of larger flows in the DPD, possibly necessitated later in the process after endothelial cell seeding, has to be studied.

Due to compaction (flattening of the leaflets in the stent) and prestrain in the leaflets, the leaflets lost coaptation after releasing them from one another, as they were (partly) grown together. This emphasizes the importance of the initial geometry, which should be optimized in future studies (a)

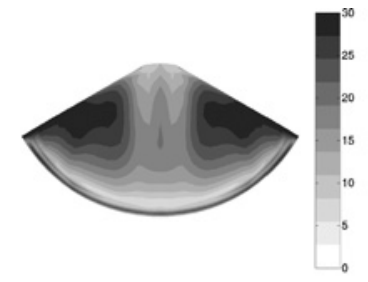

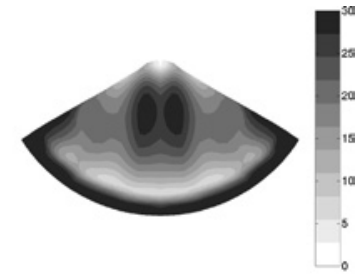

(b)

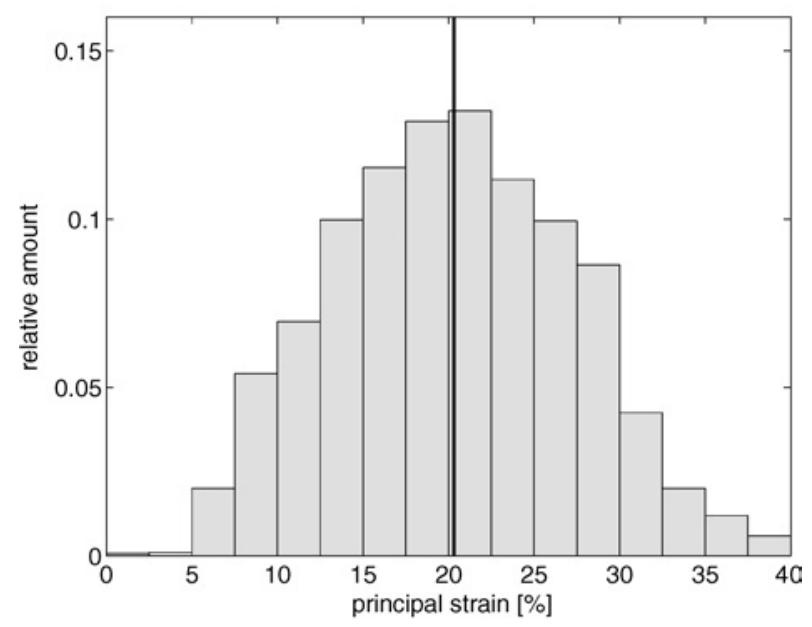

(c)

FIGURE 9. The dynamic strain distribution after 4 weeks of culturing at the upstream (a) and downstream (b) surfaces of the leaflets at an applied dynamic transvalvular pressure difference of $37 \mathrm{~mm} \mathrm{Hg}$. The grayscale represents the amount of dynamic strains in \%. The relative amount of the overall dynamic strains found in the leaflet and the mean value are shown in the histogram (c).

to retain sufficient coaptation areas after culturing. The prestrain is estimated to be in the range of $3-5 \%$ and might already be sufficient for optimized tissue formation and mechanical properties. Although no significant differences were found in mechanical properties when using additional dynamic strains, we believe that dynamic straining might further enhance tissue organization, based on the qualitative observations by histology. Enhanced tissue organization will represent itself in anisotropic mechanical properties and elucidating these properties should be the focus of future, more extensive, studies. Dynamic straining will be of even larger importance in stentless valves as the prestrain in those valves will be much less when dealing with a more pliable aortic wall. The stent used in this study was solely for experimental use and should be replaced by a tissueengineered wall for use in animal studies.

To get insight into the amount of dynamic strains applied to the leaflets, the dynamic strain distribution was estimated using numerical analyses. The mean dynamic strain within the valve leaflets cultured in this study, as an example for future use, were estimated to vary from $8 \%$ at 2 weeks to $20 \%$ 
at 4 weeks of culturing (Fig. 9 and Table 3). The estimated dynamic strains tended to increase between week 2 and 3 , while the applied dynamic transvalvular pressures was decreased, indicating loss of scaffold support. The amount of applied dynamic strains in this study might have been too large for optimized tissue formation, but application of large dynamic strains in the DPD was shown feasible. As currently the strains in the leaflets are determined afterwards, a future research focus for optimization of the Diastolic Pulse Duplicator is the development of a noninvasive method to determine tissue strains directly during culturing and to integrate this feature in a feedback loop to control the magnitude of the strains.

In the numerical analysis, an initial stress-free configuration of the leaflets is assumed, which is not completely true as the valve leaflets show compaction during culturing. In future studies, compaction behavior has to be incorporated into the model. The material properties of the leaflets are assumed to be homogeneous and isotropic, which might not be the case after a certain culture period due to the influence of prestrain in mainly the circumferential direction and the local dynamic strain distribution in the leaflets. In case of anisotropic properties, to be elucidated in future studies, an extended model should be used for the finite element analyses as described by Driessen et al. ${ }^{4}$.

In conclusion, the strain-based approach to tissue engineer human heart valve leaflets has proven its feasibility using the Diastolic Pulse Duplicator, offering new possibilities towards tissue engineering of functional human aortic heart valve replacements.

\section{ACKNOWLEDGMENTS}

Part of this work was supported by a personal grant (A. Mol) from the Netherlands Organization for Scientific Research (NWO, grant number R 90-189).

\section{REFERENCES}

${ }^{1}$ Barron, V., E. Lyons, C. Stenson-Cox, P. E. McHugh, and A. Pandit. Bioreactors for cardiovascular cell and tissue growth: A review. Ann. Biomed. Eng. 31:1017-1030, 2003.

${ }^{2}$ Chapman, G. B., W. Durante, J. D. Hellums, and A. I. Schafer. Physiological cyclic stretch causes cell cycle arrest in cultured vascular smooth muscle cells. Am. J. Physiol. Heart Circ. Physiol. 278:H748-H754, 2000.

${ }^{3}$ Dethlefsen, S. M., D. Shepro, and P. A. D'Amore. Comparison of the effects of mechanical stimulation on venous and arterial smooth muscle cells in vivo. J. Vasc. Res. 33:405-413, 1996.

${ }^{4}$ Driessen, N. J. B., C. V. C. Bouten, and F. P. T. Baaijens. A structural constitutive model for collagenous cardiovascular tissue incorporating the angular fiber distribution. J. Biomech. Eng. 127:494-503, 2005.

${ }^{5}$ Dumont, K., J. Yperman, E. Verbeken, P. Segers, B. Meuris, S. Vandenberghe, W. Flameng, and P. R. Verdonck. Design of a new pulsatile bioreactor for tissue engineered aortic heart valve formation. Artif. Organs 26:710-714, 2002.

${ }^{6}$ Engelmayr, G. C., E. Rabkin, F. W. H. Sutherland, F. J. Schoen, J. E. Mayer, and M. S. Sacks. The independent role of cyclic flexure in the early in vitro development of an engineered heart valve tissue. Biomaterials 26:175-187, 2005.

${ }^{7}$ Hildebrand, D. K., Z. J. Wu, J. E. Mayer, and M. S. Sacks. Design and hydrodynamic evaluation of a novel pulsatile bioreactor for biologically active heart valves. Ann. Biomed. Eng. 32:10391049, 2004.

${ }^{8}$ Hoerstrup, S. P., R. Sodian, S. Daebritz, J. Wang, E. A. Bacha, D. P. Martin, A. M. Moran, K. J. Guleserian, J. S. Sperling, S. Kaushal, J. P. Vacanti, F. J. Schoen, and J. E. Mayer. Functional living trileaflet heart valves grown in vitro. Circulation 102(suppl III):III-44-III-49, 2000.

${ }^{9}$ Hoerstrup, S. P., R. Sodian, J. S. Sperling, J. P. Vacanti, and J. E. Mayer. New pulsatile bioreactor for in vitro formation of tissue engineered heart valves. Tissue Eng. 6:75-79, 2000.

${ }^{10}$ Jockenhoevel, S., G. Zund, S. P. Hoerstrup, A. Schnell, and M. Turina. Cardiovascular tissue engineering: A new laminar flow chamber for in vitro improvement of mechanical tissue properties ASAIO J. 48:8-11, 2002.

${ }^{11}$ Kim, B., J. Nikolovski, J. Bonadio, and D. J. Mooney. Cyclic mechanical strain regulates the development of engineered smooth muscle tissue. Nat. Biotechnol. 17:979-983, 1999.

${ }^{12} \mathrm{Kim}$, B., and D. J. Mooney. Scaffolds for engineering smooth muscle under cyclic mechanical strain conditions. J. Biomech. Eng. 122:210-215, 2000.

${ }^{13}$ Lee, A. A., D. A. Graham, S. Dela Cruz, A. Ratcliffe, and W. J. Karlon. Fluid shear stress-induced alignment of cultured vascular smooth muscle cells. J. Biomech. Eng. 124:37-43, 2002.

${ }^{14}$ McCulloch, A. D., A. B. Harris, C. E. Sarraf, and M. Eastwood. New multi-cue bioreactor for tissue engineering of tubular cardiovascular samples under physiological conditions. Tissue Eng. 10:565-573, 2004.

${ }^{15}$ Mironov, V., V. Kasyanov, K. McAllister, S. Oliver, J. Sistino, and R. Markwald. Perfusion bioreactor for vascular tissue engineering with capacities for longitudinal stretch. J. Cranio-fac. Surg. 14:340-347, 2003.

${ }^{16} \mathrm{Mol}$, A., C. V. C. Bouten, G. Zund, C. I. Guenter, J. F. Visjager, M. I. Turina, F. P. T. Baaijens, and S. P. Hoerstrup. The relevance of large strains in functional tissue engineering of heart valves. Thorac. Cardiovasc. Surg. 51:78-83, 2003.

${ }^{17}$ Mol, A., M. I. van Lieshout, G. C. Dam-de Veen, S. Neuenschwander, S. P. Hoerstrup, F. P. T. Baaijens, and C. V. C. Bouten. Fibrin as a cell carrier in cardiovascular tissue engineering applications. Biomaterials 26:3113-3121, 2005.

${ }^{18}$ Mosmann, T. Rapid colorimetric assay for cellular growth and survival: Application to proliferation and cytotoxicity assays. J. Immunol. Methods 65:55-63, 1983.

${ }^{19}$ Narita, Y., K. Hata, H. Kagami, A. Usui, M. Ueda, and Y. Ueda. Novel pulse duplicating bioreactor system for tissueengineered vascular constructs. Tissue Eng. 10:1224-1233, 2004.

${ }^{20}$ Niklason, L. E., J. Gao, W. M. Abbott, K. K. Hirschi, S. Houser, R. Marini, and R. Langer. Functional arteries grown in vitro. Science 284:489-493, 1999.

${ }^{21}$ Niklason, L. E., W. Abott, J. Gao, B. Klagges, K. K. Hirschi, K. Ulubayram, N. Conroy, R. Jones, A. Vasanawala, S. Sanzgiri, and R. Langer. Morphological and mechanical characteristics of engineered bovine arteries. J. Vasc. Surg. 33:628-638, 2001.

${ }^{22}$ O'Callaghan, C. J., and B. Williams. Mechanical straininduced extracellular matrix production by human vascular smooth muscle cells. Hypertension 36:319-324, 2000 . 
${ }^{23}$ Rutten, M. C. M., M. Wijlaars, A. Mol, E. A. van Dam, G. J. Strijkers, K. Nicolay, and F. P. T. Baaijens. The valve exerciser: A novel bioreactor for physiological loading of tissue-engineered aortic valves. J. Biomech. (under revision).

${ }^{24}$ Schned, A. R., K. J. Wheeler, C. A. Hodorowski, J. A. Heaney, M. S. Ernstoff, R. J. Amdur, and R. D. Harris. Tissue-shrinkage correction factor in the calculation of prostate cancer volumer. Am. J. Surg. Pathol 20:1501-1506, 1996.

${ }^{25}$ Schnell, A. M., S. P. Hoerstrup, G. Zund, S. Kolb, R. Sodian, J. F. Visjager, J. Grunenfelder, A. Suter, and M. Turina. Optimal cell source for cardiovascular tissue engineering: Venous vs. aortic human myofibroblasts. Thorac. Cardiovasc. Surg. 49:221-225, 2001.

${ }^{26}$ Segal, A. SEPRAN User Manual, Standard Problems and Programmers Guide. Leidschendam: Ingenieursbureau SEPRA, the Netherlands, 1984.

${ }^{27}$ Seliktar, D., R. A. Black, R. P. Vito, and R. M. Nerem. Dynamic mechanical conditioning of collagen-gel blood vessel constructs induces remodeling in vitro. Ann. Biomed. Eng. 28:351-362, 2000.

${ }^{28}$ Sodian, R., T. Lemke, C. Fritsche, S. P. Hoerstrup, P. Fu, E. V. Potapov, H. Hausmann, and R. Hetzer. Tissue-engineering bioreactors: A new combined cell-seeding and perfusion system for vascular tissue engineering. Tissue Eng. 8:863-870, 2002.

${ }^{29}$ Stradins, P., R. Lacis, I. Ozolanta, B. Purina, V. Ose, L. Feldmane, and V. Kasyanov. Comparison of biochemical and structural properties between human aortic and pulmonary valve. Eur. J. Cardiothorac. Surg. 26:634-639, 2004.

${ }^{30}$ Thompson, C. A., P. Colon-Hernandez, I. Pomerantseva, B. D. MacNeil, B. Nasseri, J. P. Vacanti, and S. N. Oesterle. A novel pulsatile, laminar flow bioreactor for the development of tissueengineered vascular structures. Tissue Eng. 8:1083-1088, 2002.

${ }^{31}$ Thubrikar, M. The Aortic Valve. Boca Raton: CRC Press, 1990 $221 \mathrm{pp}$.

${ }^{32}$ Ueba, H., M. Kawakami, and T. Yaginuma. Shear stress as an inhibitor of vascular smooth muscle cell proliferation. Arterioscler. Thromb. Vasc. Biol. 17:1512-1516, 1997.

${ }^{33}$ Watase, M., M. A. Awolesi, J. Ricotta, and B. E. Sumpio. Effect of pressure on cultured smooth muscle cells. Life Sci. 61:987996, 1997.

${ }^{34}$ Williams, C., and T. M. Wick. Perfusion bioreactor for small diameter tissue-engineered arteries. Tissue Eng. 10:930-941, 2004. 